\title{
ADAPTACIÓN Y FIABILIDAD DEL CUESTIONARIO DE RED SOCIAL, VERSIÓN ESPAÑOLA DEL «QUESTIONARIO SULLA RETE SOCIALE», APLICADO A PERSONAS CON TRASTORNO MENTAL SEVERO
}

SPANISH VERSION AND RELIABILITY OF THE «SOCIAL NETWORK QUESTIONNAIRE», APPLIED TO PEOPLE WITH SEVERE MENTAL ILLNESS

\section{RESUMEN}

Introducción: El objetivo de este artículo es presentar la adaptación española del «Questionario Sulla Rete Sociale» -SNQ- (Cuestionario de Red Social -CURES-), cuya fiabilidad se ha analizado en una muestra de personas con trastorno mental severo de Andalucía (España).

Metodología: Se tradujo el cuestionario al español y se valoró la fiabilidad entre jueces y la consistencia entre instantes en una muestra de 30 sujetos residentes en alojamientos protegidos. Posteriormente se evaluó la consistencia interna a partir de las respuestas de 225 sujetos de similares características. Para el análisis estadístico se utilizaron los Coeficientes Correlación Intraclase y Alfa de Cronbach.

Resultados: Fiabilidad entre jueces: Intraclase entre 0.716 y 1.000; Consistencia entre instantes: Intraclase entre $0.412 \mathrm{y}$ 1.000; Consistencia Interna: Alfa $=0.723$.

Conclusiones: La versión española del SNQ (CURES) es un instrumento de medida que presenta niveles razonables de fiabilidad para evaluar la red social de personas con trastorno mental severo en estructuras residenciales.

Palabras clave: fiabilidad, evaluación red social, trastorno mental severo.

\section{ABSTRACT}

Introduction: The aims of this paper is to present the Spanish version of the Social Network Questionnaire -SNQ- and its reliability that has been analysed on a sample of people with severe mental illness in Andalusia (Spain).

Method: The questionnaire was translated into Spanish and its inter-rate and test-retest agreement were assessed in a sample of 30 subjects, residents in different sheltered homes. Afterwards, the internal consistency was assessed using the answerers of 225 subjects of similar characteristics. For the statistical analysis Intraclass and Cronbach coefficients were used.

Results: Inter-rate agreement: Intraclass from 0.716 to 1.000 ; Test-retest agreement: Intraclass from 0.412 to 1.000 ; Internal Consistency: Alfa $=0.723$.

Conclusion: The Spanish version of the SNQ (CURES) is an assessment instrument with reasonable reliability to assess the social network of people with severe mental illness in residential facilities.

Key words: reliability, social network assessment, severe mental illness. 


\section{INTRODUCCIÓN}

El estudio de las relaciones sociales de las personas es reconocido desde hace tiempo como un campo prometedor dentro de la epidemiología, en la medida en que constituyen un importante factor de protección frente a distintos tipos de problemas y patologías tanto biológicas como psíquicas (1-4). En concreto hay evidencia creciente con respecto al papel que el número, frecuencia, diversidad y calidad de los contactos sociales, lo que suele resumirse bajo el concepto de «redes sociales», parece tener, tanto para asegurar la estabilidad sintomatológica como para una satisfactoria y activa permanencia en la comunidad de personas con enfermedades mentales graves $(1,4-5)$.

Como datos relevantes que se desprenden de distintos estudios y revisiones del tema, parece habitual que las personas con este tipo de problemas suelen tener redes de menor tamaño y diversidad, restringiéndose sus contactos sociales habituales a pocas personas, habitualmente localizadas en el ámbito familiar o en el propio contexto de los cuidados sanitarios y sociales que reciben $(2,4,6)$. En este sentido, un campo específico de evaluación es el referido a las redes sociales de usuarios de programas residenciales (5).

Por ello, dentro del estudio emprendido en Andalucía sobre el programa residencial de FAISEM ${ }^{1}$, se consideró imprescindible algún tipo de exploración de los contactos sociales habituales de las personas que residían en algunos de los dispositivos del programa $(1,7)$.

Como muchos otros campos de la investigación sobre factores psicosociales, el estudio de las relaciones sociales se enfrenta a dificultades metodológicas específicas, que tienen que ver con la utilización de conceptos operativos y de sus correspondientes instrumentos de medida. En lo que respecta al primer aspecto tiene especial trascendencia el mencionado concepto de «red social», que intenta delimitar dimensiones medibles de cara a su investigación cuantitativa. De hecho las publicaciones de mayor interés, a las que hemos hecho referencia anteriormente (2-6), hablan específicamente de redes sociales, entendiendo por tales el número y frecuencia de contactos con personas concretas en las que entran los sujetos en estudio. Aspecto que deja fuera dimensiones menos fácilmente medibles como la tipología de contactos y las vivencias subjetivas que producen. Aún así la evidencia acumulada es suficientemente significativa como para tener en cuenta esta dimensión cuantitativa.

En relación con los instrumentos, no hay un consenso razonable al respecto, encontrándonos frecuentemente con un uso habitual de cuestionarios y escalas específicamente diseñados para cada investigación (2-4). Por ello, al buscar un instrumento concreto para nuestro estudio las opciones se redujeron básicamente a tres. En primer lugar el cuestionario utilizado en los estudios del TAPS (8), de los que provienen la

1. «Evaluación del Programa Residencial para Enfermos Mentales en Andalucía», cofinanciado por el Fondo de Investigación Sanitaria (Exp. $n^{\circ}$ 00/0973) y FAISEM 
mayoría de los instrumentos utilizados en el nuestro. Sin embargo, la experiencia anterior de uso del instrumento mostró algunas dificultades que derivan básicamente de su complejidad, dificultades que nos parecieron adecuadamente contrarrestadas por su utilidad real. Del mismo modo se desechó la entrevista Mannhein (9-10), sobre la que también había cierta experiencia en el grupo investigador. El conocimiento del trabajo del grupo italiano coordinado por Lorenza Magliano, que acababa de hacer públicos los resultados de una importante investigación sobre las familias de personas con esquizofrenia (11), nos facilitó un instrumento que nos pareció razonablemente adecuado por sus características básicas: contenido, propiedades psicométricas y facilidad, a pesar de que su empleo original se realizó en familias y no en personas con enfermedad mental grave.

Por ello tras consultar con la autora la pertinencia de esa nueva utilización, se realizó la traducción y adaptación española del cuestionario, abordándose el análisis de la fiabilidad en una muestra de personas con trastorno mental severo, residente en dispositivos del programa residencial de la Fundación Andaluza para la Integración Social del Enfermo Mental (12). El artículo presenta las características generales de la versión española del instrumento y los resultados del estudio de su fiabilidad.

\section{- Cuestionario de Red Social -CURES-}

La versión original del Questionario Sulla Rete Sociale»-SNQ-, fue elaborada como ya se ha mencionado por L. Magliano para una investigación sobre carga familiar en el cuidado de pacientes con esquizofrenia, en cinco países europeos (11). Existe una segunda versión, en inglés, para su utilización en el citado proyecto. El instrumento consta de 15 preguntas y mide las características de la red social de cada persona, agrupándolas en cuatro factores: 1) calidad y frecuencia de los contactos sociales 2) apoyo social practico 3) apoyo afectivo y 4) compañero de apoyo.

La consistencia entre instantes del cuestionario original se calculó con una muestra de 50 familiares, aplicándoseles el mismo cuestionario diez días más tarde. El Coeficiente Kappa de Cohen (13) osciló entre 0.50 y 1 para el $69 \%$ de los items. La consistencia interna se midió con el Coeficiente Alfa de Cronbach (14) obteniéndose un alfa para cada uno de los factores: 1) 0.68 ; 2) 0.75 ; 3) 0.66 ; 4) 0.56 ; siendo el alfa global 0.66 .

La versión española (anexo I) consta de 18 preguntas, 3 más que la versión original, las cuales se añadieron para su aplicación en el mencionado estudio aunque no se sometieron a la validación (las preguntas 17 y 18 relacionadas con el peso de las relaciones internas al programa residencial -compañeros y personal- dentro de la red social y la pregunta 16 , que solo se utiliza si más del $50 \%$ de las respuestas obtienen una puntuación de 8 o 9, motivo por el cual no se analiza la información aportada por el usuario). En la mayoría de los items, las puntuaciones 7,8 y 9 , que se sitúan fuera de la escala ordinal, hacen referencia a las categorías de «No aplicable», «No hay respuesta» y «Respuesta no clasificable» respectivamente. La información proviene del propio residente, recogida por un encuestador entrenado. 


\section{METODOLOGÍA}

\section{- Sujetos}

Para el estudio de fiabilidad entre jueces y entre instantes se utilizó una muestra de 30 usuarios alojados en dispositivos residenciales específicos de FAISEM, con diagnóstico de enfermedad mental severa de larga evolución.

Finalmente, para el estudio de la consistencia interna se utilizaron 225 usuarios con diagnósticos similares al grupo anterior, los cuales habían cumplimentado el cuestionario en su totalidad en el marco del mencionado estudio $(1,7)$. Ver en tabla I las características sociodemográficas de ambas muestras.

\section{- Procedimiento}

El citado cuestionario fue traducido al castellano a partir de la versión original italiana, comparándola con la versión inglesa traducida por los propios autores para su uso en países de habla anglosajona. Posteriormente se procedió a contrastar ambas traducciones, siendo revisada por varios profesionales de salud mental para detectar posibles incoherencias entre la versión española y la original e identificar aspectos que pudieran plantear dificultades de comprensión en la población española.

Sobre la traducción se realizaron algunos cambios, motivados principalmente porque la versión original iba dirigida a familiares de manera autoaplicada, mientras que en el citado proyecto el cuestionario va dirigido a los propios usuarios, administrado por un entrevistador. Esta tarea se llevó a cabo en varias sesiones, en las cuales se discutieron diferentes alternativas, eligiéndose las más adecuada para la finalidad del estudio.

$Y$ finalmente, la traducción fue revisada por un grupo de expertos (constituidos por profesionales de salud mental y de servicios sociales) para corroborar su validez de contenido para su aplicación a pacientes.

En dicho proceso se detectó que las preguntas que hacían referencia al $2^{\circ}$ factor (Apoyo social Práctico) no eran pertinentes para la población en estudio, dado que una de las características del Programa Residencial es la de dar cobertura a las necesidades básicas de los usuarios, entre las cuales se encuentran las de apoyo práctico. De hecho, el $42 \%$ de los usuarios entrevistados no las contestaron.

Posteriormente, se procedió a su validación. El estudio de las cualidades del instrumento se llevó a cabo en dos vertientes: fiabilidad entre jueces y entre instantes y consistencia interna del conjunto de la escala y de cada una de las subescalas,

Para el estudio de fiabilidad (entre jueces y entre instantes), un solo encuestador entrenado entrevistó en un primer momento a los integrantes de la $1^{\text {a }}$ muestra ( $\left.\mathrm{n}: 30\right)$.

La fiabilidad entre jueces, se realizó con la ayuda de un segundo evaluador debidamente entrenado, que sin intervenir de manera activa en las entrevistas anteriores, observaba y puntuaba de manera independiente las respuestas emitidas por los usuarios. 
Para calcular la consistencia entre instantes, el mismo encuestador volvió a entrevistar a 27 de los 30 usuarios 45 días más tarde. Se había producido un fallecimiento tras las primeras entrevistas y dos de las personas a encuestar por segunda vez no quisieron responder a las preguntas.

En relación a la consistencia interna, cuatro encuestadores debidamente entrenados entrevistaron a los integrantes de la $2^{\text {a }}$ muestra ( $\mathrm{n}$ : 225), la cual se había dividido en cuatro grupos, siguiendo criterios geográficos y de homogeneidad en el tamaño de los mismos.

\section{- Método Estadístico}

Para medir la concordancia entre observadores y entre instantes ítem a ítem, salvo los incluidos en la segunda subescala que no fue considerada por las razones ya expuestas, dado que las contestaciones a los ítems eran ordinales, se empleó como coeficiente de concordancia el coeficiente de correlación intraclase, así como su intervalo de confianza 15. Para el estudio de la consistencia interna se empleó el alfa de Cronbach 14 con su correspondiente intervalo de confianza para el conjunto de la escala y para las subescalas 1,3 y 4 del cuestionario original. Tal como vimos con anterioridad, el primer objetivo se cubrió empleando una muestra de 30 usuarios y el segundo con una muestra de 225 usuarios, los cuales habían contestado todos los ítems del cuestionario. El paquete estadístico empleado fue el SPSS 12.0.

\section{RESULTADOS}

En relación a la fiabilidad entre jueces de los items que se consideraron pertinente, incluidos en la versión original $(1,2,3,4,5,9,10,11,12,13$ y 14), el coeficiente de correlación intraclase se sitúa entre 0.716 y 1.000, obteniendo un valor por encima de 0.70 el $100 \%$ de los ítems (tabla II).

En cuanto a la consistencia entre instantes, el coeficiente de correlación intraclase se sitúa entre 0.412 y 1.000 , obteniendo un valor por encima de 0.50 el $75 \%$ de los ítems (tabla III).

En relación a la consistencia interna, se obtuvo un valor medio de alfa de 0.723 , con un intervalo de confianza de $0.667-0.774$. En cuanto a los factores, el valor medio obtenido para el primero fue de $0.511 ; 0.643$ para el tercero y 0.654 para el cuarto (tabla IV). 


\section{CONCLUSIONES}

Los resultados del análisis de fiabilidad entre jueces ${ }^{2}$ de los distintos items del cuestionario una vez excluidos los items del segundo factor, indican un buen nivel de concordancia entre evaluadores (el $100 \%$ de los items se sitúan por encima de 0.70 y un $58 \%$ por encima de 0.90 -coeficiente de correlación intraclase-), siendo un instrumento que parece recoger la situación del usuario de manera fiable independientemente del observador, siempre que éste esté convenientemente entrenado.

En relación a la consistencia entre instantes, un coeficiente de correlación intraclase por encima de 0.50 en el $75 \%$ de los items y por encima de 0.70 en el 58 $\%$, indican un nivel de acuerdo moderado, que son parecidos a los obtenidos por los autores de la versión original (11), pese a que allí se usó como coeficiente de concordancia el estadístico Kappa (13) (entre 0.50 y 1 para el $69 \%$ de los ítems) y que el periodo entre ambas mediciones diferían en 35 días, aunque esta última diferencia no parece tener demasiada relevancia.

El hecho de que en la mayoría de las preguntas se solicitara información respecto a un periodo de tiempo determinado, puede haber influido en las respuestas de los sujetos tras haber transcurrido 45 días desde la primera medición. Adicionalmente, en la valoración que hace el usuario sobre sus relaciones sociales puede influir la situación psicopatológica que atraviese el mismo en el momento de la entrevista, siendo ello una posible limitación de la repetibilidad del cuestionario.

En relación al análisis de la correlación entre los distintos items del cuestionario, los resultados indican una consistencia interna aceptable $(0.667-0.774)$, siendo éste ligeramente superior a los obtenidos por los autores de la versión original 11 con un valor medio de alfa de 0.66 , si bien hay que tener en cuenta que la población objeto en nuestro caso fueron usuarios, mientras que el cuestionario original estaba pensado para familiares.

Sin embargo, al analizar la consistencia interna por cada uno de los tres factores restantes, los resultados indican una correlación intraclase ligeramente inferior en los factores primero y tercero (Calidad y frecuencia de los contactos sociales y Apoyo afectivo), aunque en relación al cuarto (Compañero de apoyo), la correlación es ligeramente superior. Hay que tener en cuenta aquí, que los items de este último factor tienen valores con escasa variabilidad (la mayoría de los usuarios de la muestra no tienen pareja).

En relación al primer factor, destacar que si se elimina el item 14, la consistencia interna del factor en cuestión aumenta de manera considerable. Suponemos que puede haber dos causas que están debajo de este hecho; una, que dicho item se encuentra separado del resto de los componentes del citado factor, y otra, que pensamos más relevante, que la formulación de la pregunta es diferente, pudiendo presentar alguna dificultad de comprensión.

2. No realizada en el estudio de validación del instrumento original 11 
Por otro lado, el hecho de que sólo se emplearan los individuos en los que el cuestionario estaba completo, puede estar sesgando al alza los valores de los coeficientes obtenidos; la razón es que los datos faltantes provienen en la mayoría de los casos de contestaciones no claras y sobre todo de la no aplicabilidad de la pregunta, por lo que los que se analizan, aún siendo una muestra muy amplia, son aquellos en los que la información ha sido aplicable y de suficiente calidad como para ser recogida, es decir aquellos en los que ha habido contestaciones más «coherentes» abundando este hecho en establecer correlaciones mayores de las que posiblemente haya.

En referencia al segundo factor, si bien en el presente estudio se ha eliminado por las razones anteriormente expuestas, sería conveniente incluirlo si las características de futuras poblaciones objeto así lo aconsejan (p.e. pacientes que residen en su propio domicilio o de sus familiares), una vez realizado el correspondiente estudio de fiabilidad.

Asumiendo los efectos que se hayan producido por el hecho de modificar la población objeto a la que iba dirigida el instrumento, pensamos que merecía la pena su utilización una vez adaptado, por su ya comentada validez de contenido y fácil aplicación.

Teniendo en cuenta lo anteriormente expuesto, el CURES aparece como un instrumento de medida que, en su adaptación española, presenta propiedades de fiabilidad aceptables para evaluar la red social de personas con trastorno mental severo, usuarios de estructuras residenciales, si bien sería conveniente futuras revisiones con la finalidad de mejorar las propiedades psicométricas de dicho instrumento. 
Tabla I

Características sociodemográficas de las muestras

\begin{tabular}{|c|c|c|c|}
\hline & & $\begin{array}{c}\text { Muestra } 1 \\
\text { (entre jueces/ } \\
\text { instantes)Muestra } 1 \\
\text { (Consistencia interna) }\end{array}$ & $\begin{array}{c}\text { Muestra } 2 \\
\text { (Consistencia } \\
\text { interna)Muestra } 2 \\
\text { (entre jueces/instantes) }\end{array}$ \\
\hline$n$ & & 30225 & 22530 \\
\hline \multirow{4}{*}{ Edad } & Edad media: & 44,1 años 45,3 años & 45,3 años 44,1 años \\
\hline & < 35 años: & $14 \% 12 \%$ & $12 \% 14 \%$ \\
\hline & 35 - 50 años: & $69 \% 57 \%$ & $57 \% 69 \%$ \\
\hline & > 50 años: & $17 \% 31 \%$ & $31 \% 17 \%$ \\
\hline \multirow{2}{*}{ Sexo } & Hombre: & $83 \% 76 \%$ & $76 \% 83 \%$ \\
\hline & Mujer: & $17 \% 24 \%$ & $24 \% 17 \%$ \\
\hline \multirow{3}{*}{ Estado civil } & Soltero: & $80 \% 84 \%$ & $84 \% 80 \%$ \\
\hline & Casado / con pareja de hecho: & $3 \% 5 \%$ & $5 \% 3 \%$ \\
\hline & Separado, divorciado, viudo: & $17 \% 11 \%$ & $11 \% 17 \%$ \\
\hline \multirow{5}{*}{$\begin{array}{l}\text { Nivel } \\
\text { educativo }\end{array}$} & No sabe leer ni escribir: & $-4 \%$ & $4 \%-$ \\
\hline & Sabe leer y escribir sin estudios: & $24 \% 31 \%$ & $31 \% 24 \%$ \\
\hline & Estudios primarios, EGB, Bachiller elemental: & $59 \% 45 \%$ & $45 \% 59 \%$ \\
\hline & Bachiller superior, FP o similar: & $14 \% 15 \%$ & $15 \% 14 \%$ \\
\hline & Estudios universitarios: & $3 \% 5 \%$ & $5 \% 3 \%$ \\
\hline \multirow{3}{*}{$\begin{array}{l}\text { Diagnóstico } \\
\text { CIE-10 }\end{array}$} & $\begin{array}{l}\text { F2 (Esquizofrenia, trastornos esquizotípicos y } \\
\text { trastornos de ideas delirantes): }\end{array}$ & $\begin{array}{l}93 \% \\
86 \%\end{array}$ & $\begin{array}{l}86 \% \\
93 \%\end{array}$ \\
\hline & F3 (Trastornos del humor): & $-6 \%$ & $6 \%-$ \\
\hline & F0-F1-F6-F7: & $7 \% 8 \%$ & $8 \% 7 \%$ \\
\hline $\begin{array}{l}\text { Evolución de } \\
\text { la enfermedad }\end{array}$ & Duración media: & 18,7 años21,9 años & 21,9 años18,7 años \\
\hline $\begin{array}{l}\text { Estancia en } \\
\text { el Programa } \\
\text { Residencial }\end{array}$ & Estancia media: & $\begin{array}{l}3,2 \text { años } \\
4,2 \text { años }\end{array}$ & $\begin{array}{l}4,2 \text { años } \\
3,2 \text { años }\end{array}$ \\
\hline
\end{tabular}


ADAPTACIÓN Y FIABILIDAD DEL CUESTIONARIO...

Tabla II

Fiabilidad entre jueces

\begin{tabular}{|c|c|c|c|c|}
\hline \multirow{2}{*}{ Item } & \multirow{2}{*}{$\mathrm{N}$} & $\begin{array}{c}\text { Correlación } \\
\text { Intraclase }\end{array}$ & \multicolumn{2}{|c|}{ Intervalo de confianza } \\
\cline { 4 - 5 } & & 0.804 & 0.625 & Superior \\
\hline 1 & 29 & 0.716 & 0.479 & 0.903 \\
\hline 2 & 30 & 0.936 & 0.866 & 0.854 \\
\hline 3 & 28 & 0.918 & 0.818 & 0.970 \\
\hline 4 & 23 & 0.841 & 0.679 & 0.964 \\
\hline 5 & 26 & 0.914 & 0.725 & 0.925 \\
\hline 9 & 28 & 1.000 & & 0.966 \\
\hline 10 & 30 & 1.000 & & \\
\hline 11 & 30 & 1.000 & 0.974 & \\
\hline 12 & 29 & 0.988 & 0.804 & 0.995 \\
\hline 13 & 26 & 0.903 & 0.701 & 0.953 \\
\hline 14 & 29 & 0.853 & & \\
\hline 15 & 26 & & & \\
\hline
\end{tabular}

Tabla III

Consistencia Fiabilidad entre instantes

\begin{tabular}{|c|c|c|c|c|}
\hline \multirow{2}{*}{ Item } & \multirow{2}{*}{$N$} & \multirow{2}{*}{$\begin{array}{c}\text { Correlación } \\
\text { Intraclase }\end{array}$} & \multicolumn{2}{|c|}{ Intervalo de confianza } \\
\cline { 4 - 5 } & & 0.795 & inferior & superior \\
\hline 1 & 26 & 0.656 & 0.592 & 0.903 \\
\hline 2 & 27 & 0.485 & 0.373 & 0.827 \\
\hline 3 & 25 & 0.650 & 0.111 & 0.737 \\
\hline 4 & 17 & 0.712 & 0.278 & 0.855 \\
\hline 5 & 23 & 0.870 & 0.158 & 0.894 \\
\hline 9 & 26 & 1.000 & 0.732 & 0.939 \\
\hline 10 & 26 & 1.000 & & \\
\hline 11 & 26 & 0.768 & & 0.852 \\
\hline 12 & 26 & 0.754 & 0.476 & 0.888 \\
\hline 13 & 26 & 0.503 & 0.152 & 0.741 \\
\hline 14 & 26 & 0.412 & 0.012 & 0.696 \\
\hline 15 & 24 & & & \\
\hline
\end{tabular}


Tabla IV

Consistencia Interna

\begin{tabular}{|c|c|c|c|c|}
\hline Item & $\begin{array}{c}\text { Escala global } \\
\text { alfa si se elimina }\end{array}$ & $\begin{array}{c}\text { Factor } 1 \\
\text { alfa si se elimina } \\
\end{array}$ & $\begin{array}{c}\text { Factor } 3 \\
\text { alfa si se elimina } \\
\end{array}$ & $\begin{array}{c}\text { Factor } 4 \\
\text { alfa si se elimina } \\
\end{array}$ \\
\hline 1 & 0.712 & 0.418 & & \\
\hline 2 & 0.702 & 0.338 & & \\
\hline 3 & 0.695 & 0.409 & & \\
\hline 4 & 0.702 & & 0.576 & \\
\hline 5 & 0.688 & & 0.587 & \\
\hline 9 & 0.679 & & 0.553 & \\
\hline 10 & 0.716 & & & * \\
\hline 11 & 0.721 & & & * \\
\hline 12 & 0.717 & & 0.640 & \\
\hline 13 & 0.687 & & 0.589 & \\
\hline 14 & 0.718 & 0.616 & & \\
\hline GLOBAL & $0.723(0.667-0.774)$ & $0.511(0.397-0.607)$ & $0.643(0.563-0.712)$ & $0.654(0.550-0.734)$ \\
\hline
\end{tabular}

* no se puede calcular porque cuando se elimina queda un único item.

\section{BIBLIOGRAFÍA}

1. López M, García-Cubillana P, Laviana M, Fernández M, Fernández L y Maestro JC. Evaluación del programa residencial para personas con trastorno mental severo en Andalucía (IV): perfiles funcionales y redes sociales de los residentes. Rehabil psicosoc 2005 (en prensa).

2. Albert M, Becker T, Mccrone P y Thornicroft G. Social network and mental health service utilisation. A literature review. Int J Soc Psychiatry 1998; 44 (4): 248-266.

3. Berkman LF y Glass T. Social integration, social networks, social support and Health. En: Berkman LF y Kawachi I. Social Epidemiology. New York: Oxford University Press, 2000; p.137-173.

4. Randolph ET. Social networks and schizophrenia. En: Mueser KT y Tarrier N. Handbook of social functioning in schizophrenia. Boston: Alyn \& Bacon, 1998; p.238-246.

5. Fakhoury WK, Murray A, Shepherd G y Priebe S. Research in supported housing. Soc Psychiatry Psychiatr Epidemiol 2002; 37: 301-315.

6. Becker T; Albert M; Angermeyer MC; Thornicroft G. Social network and service utilisation in patients with severe mental illness. En: Tansella M, ed. Making rational mental health services. Roma: Pensioro Scientifico Editore, 1997; p.113-125.

7. López M, Laviana M, García-Cubillana P, Fernández L, Moreno B y Maestro JC. Evaluación del programa residencial para personas con trastorno mental severo en Andalucía (I): Descripción general del programa y del estudio. Rehabil psicosoc. 2005; 2 (1): 2-15. 
8. Leff J, 0’Driscoll C, Dayson D, Wills W y Anderson J. The TAPS Proyect. 5: The Structure of Social-Network Data Obtained from Long-Stay Patients. Br J Psychiatry 1990; 157: 848-852.

9. Veiel HO. The Mannheim Interview on Social Support. Reliability and validity data from three samples. Soc Psychiatry Psychiatr Epidemiol 1990; 25 (5): 250-9.

10. Vazquez AJ, Garcia RJ. The Mannheim interview on social support psychometric characteristics of a Spanish version. Soc Psychiatry Psychiatr Epidemiol 1997; 32 (4): 208-21.

11. Magliano L, Fadden G, Madianos M, Caldas de Almeida JM, Held T, Guarneri M, Marasco C, Tosini P, Maj M. Burden on the families of patients with schizophrenia: results of the BIOMED I study. Soc Psychiatry Psychiatr Epidemiol 1998; 33: 405-412.

12. Lara L; López M. La provisión de recursos de apoyo social en Andalucía. En: García J; Espino A; Lara L (Eds.) La psiquiatría en la España de fin de siglo. Madrid: Díaz de Santos, 1998; p.213-224.

13. Cohen J: A coefficient of agreement for nominal scales. Educ Psychol Meas 1960; 20: $37-46$.

14. Cronbach LJ: Coefficient alpha and the internal structure of test. Psychometrika 1951; 16: 297-334

15. Martín A y Luna del Castillo JD. Bioestadística para las Ciencias de la Salud. Madrid: Ediciones Norma-Capitel, 2004.

\section{ANEXO I: CUESTIONARIO DE RED SOCIAL -CURES-}

\section{- Instrucciones}

El cuestionario se pasa al usuario directamente. Las preguntas deben hacerse de un modo abierto y explicarse todo lo que sea necesario para obtener una respuesta. Cumplimentar cada respuesta con el número correspondiente.

Una respuesta se considera no clasificable cuando no incluye suficiente información para evaluar con exactitud su contenido. Las respuestas no clasificables incluyen los «no sé», el material alucinatorio y/o delirante, etc.

Una pregunta se considera no aplicable cuando no es pertinente formularla al entrevistado.

En el caso de usuarios que, en el momento de la entrevista presenten alguna sintomatología que dificulte la realización de la misma, preguntar al personal del alojamiento para intentar diferenciar si se trata de una situación habitual o de una afectación temporal. En este último caso, la entrevista podrá volver a intentarse en los 30 días siguientes.

Toda la información será estrictamente confidencial y estará regulada por los códigos de ética profesional 


\section{- Cuestionario}

1) ¿En los últimos dos meses, has llamado o te has visto con amigos o familiares que no viven contigo?

1. Nunca

2. A veces

3. Frecuentemente

4. Todos los días

8. No hay respuesta

9. Respuesta no clasificable

2) ¿En los últimos dos meses, amigos y familiares que no viven contigo han llamado o han venido a verte?

1. Nunca

2. A veces

3. Frecuentemente

4. Todos los días

8. No hay respuesta

9. Respuesta no clasificable

3) ¿En los últimos dos meses, amigos y familiares que no viven contigo te han hablado sobre sus propios problemas?

1. Nunca

2. A veces

3. Frecuentemente

4. Todos los días

8. No hay respuesta

9. Respuesta no clasificable

4) ¿En los dos últimos meses, cuando te has sentido nervioso o preocupado, has tenido a alguien que te ha tranquilizado?

1. Nunca

2. A veces

3. Frecuentemente

4. La mayor parte de las veces

7. No aplicable, nunca te has sentido nervioso ni preocupado

8. No hay respuesta

9. Respuesta no clasificable 
5) ¿En los dos últimos dos meses, cuando te ha ocurrido algo bueno, has tenido alguien con quien comentarlo?

1. Nunca

2. A veces

3. Frecuentemente

4. La mayor parte de las veces

7. No aplicable, nunca te ha ocurrido nada bueno

8. No hay respuesta

9. Respuesta no clasificable

6) ¿En los dos últimos meses, si hubieras estado enfermo, habrías tenido a alguien que te cuidara?

1. No

2. Posiblemente

3. Muy probablemente

4. Seguro

8. No hay respuesta

9. Respuesta no clasificable

7) ¿En los dos últimos meses, cuando has necesitado ayuda en las tareas o reparaciones domésticas, has encontrado fácilmente a alguien que te echara una mano?

1. Nunca

2. A veces

3. Frecuentemente

4. La mayor parte de las veces

7. No aplicable, nunca has necesitado ayuda con los quehaceres domésticos

8. No hay respuesta

9. Respuesta no clasificable

8) ¿En los dos últimos meses, has encontrado fácilmente a alguien que te ha hecho los recados cuando no has podido hacerlos?

1. Nunca

2. A veces

3. Frecuentemente

4. La mayor parte de las veces

7. No aplicable, nunca has necesitado ayuda

8. No hay respuesta

9. Respuesta no clasificable 
9) ¿En los últimos dos meses, has tenido a un amigo o familiar de confianza para hablarle de tus propios problemas?

1. Nunca

2. A veces

3. Frecuentemente

4. La mayor parte de las veces

8. No hay respuesta

9. Respuesta no clasificable

10) ¿Actualmente tienes novia/o o estás saliendo con alguna persona de manera regular?

1. No

4. Sí

7. No aplicable (ha contestado «Sí» a la pregunta anterior)

8. No hay respuesta

9. Respuesta no clasificable

Si la respuesta a la pregunta anterior es «Sì»:

11) ¿Vives en la actualidad con tu pareja?
1. No
4. Sí
8. No hay respuesta
9. Respuesta no clasificable

12) ¿Cuántos amigos de confianza tienes? (responder a continuación a la pregunta 17)
1. Ninguno
2. 1
3. 2 ó 3
4. 4 ó más
8. No hay respuesta
9. Respuesta no clasificable

13) ¿A cuántos familiares consideras como amigos de confianza?
1. Ninguno
2. 1
3. 2 ó 3
4. 4 ó más
8. No hay respuesta
9. Respuesta no clasificable 
14) ¿En los dos últimos meses, te has sentido parte de un grupo (asociación cultural, religiosa, recreativa o grupo de familiares, entre otros), y ello ha sido importante en tu vida?

1. No

4. Sí

8. No hay respuesta

9. Respuesta no clasificable

15) Comparando con hace un año, tus relaciones sociales se han...

1. Empeorado

2. Mantenido igual

3. Mejorado

4. Mejorado mucho

8. No hay respuesta

9. Respuesta no clasificable

16) En el caso de que la mayoría de las respuestas hayan sido «No hay respuesta» 0 «Respuesta no clasificable» (más del $50 \%$ ), sería por:

1. No quiere responder

2. Lenguaje incoherente

3. Desconocimiento del castellano

4. Mutismo

5. Otras razones (especificar)

7. No aplicable (las respuestas «No hay respuesta» 0 «Respuesta no clasificable» no superan el $50 \%$ )

Información adicional para el Programa Residencial

17) De las personas que consideras «amigos de confianza», ¿cuáles de ellas pertenecen al programa residencial (incluyendo usuarios y profesionales)?

1. Ninguna

2. Una minoría

3. La mitad

4. La mayoría

5. Todas

7. No aplicable, no tienes amigos de confianza

8. No hay respuesta

9. Respuesta no clasificable

Preguntar al personal cuidador: 
18) Del total de personas con las que el usuario mantiene relaciones habituales (llamadas, visitas, personas a las que consulta o con las que habla regularmente), ¿cuáles de ellas pertenecen al programa residencial (incluyendo usuarios y profesionales)?

1. Ninguna

2. Una minoría

3. La mitad

4. La mayoría

5. Todas

8. No hay respuesta

9. Respuesta no clasificable

Pablo García-Cubillana

Fundación Andaluza para la Integración Social del Enfermo mental. Sevilla.

Juan de Dios Luna

Departamento de Bioestadística, Universidad de Granada.

Margarita Laviana

Área de Salud Mental Hospital "Virgen del Rocio", SAS, Sevilla.

Grupo Andaluz de Investigación en Salud Mental.

Luís Fernández

Fundación Andaluza para la Integración Social del Enfermo mental. Sevilla.

David Perdiguero

Fundación Andaluza para la Integración Social del Enfermo mental. Sevilla.

Marcelino López

Fundación Andaluza para la Integración Social del Enfermo mental. Sevilla.

Dirección correspondencia:

Pablo García-Cubillana

Fundación Andaluza para la Integración Social del Enfermo mental.

Avda. Las Ciencias, 27, Acc. A

41020 Sevilla

E-mail: pablo.garciacubillana@juntadeandalucia.es 